\title{
Migraine-Induced Epistaxis and Sporadic Hemiplegic Migraine: Unusual Features in the Same Patient
}

\author{
José Barros ${ }^{a, b}$ Joana Damásio ${ }^{a} \quad$ Assunção Tuna $^{a}$ \\ José Pereira-Monteiro ${ }^{a, b}$ \\ ${ }^{a}$ Hospital de Santo António, Centro Hospitalar do Porto, and ${ }^{\mathrm{b}}$ Instituto de \\ Ciências Biomédicas Abel Salazar, Universidade do Porto, Porto, Portugal
}

\section{Key Words}

Epistaxis - Migraine with aura $\cdot$ Sporadic hemiplegic migraine $\cdot$ Spreading depression

\begin{abstract}
Background: Since the mid-19th century, epistaxis and migraine have been occasionally associated with each other. Nevertheless, we found only two cases in the contemporary medical literature. Sporadic hemiplegic migraine is a subtype of migraine with reversible motor deficits, without similar episodes in relatives.
\end{abstract}

Case: We describe a 47-year-old male with a history of migraine with a scintillating scotoma starting at the age of 20 . In some of the episodes, he developed epistaxis in the resolution phase of migraine. At the age of 35 , he experienced a visual aura followed by transient aphasia, left crural weakness and headache. Contralateral similar episodes occurred in the subsequent months. Neurological examination and MRI were normal. Mutations in CACNA1A, ATP1A2, SCN1A and NOTCH3 were excluded.

Discussion: Three distinct aspects deserve our consideration. This is the first report of migraine-induced epistaxis involving aura; the scarcity of similar reports may be due to the lack of a guided anamnesis. The complex aura presented had a peculiar topography, inconsistent with the classical analytical neurological semiology. This may suggest that the spreading depression affects the brain bilaterally but in an uneven and elective manner. Lastly, the present report conveys that the late appearance of complex auras requires improbable interactions between environmental and endogenous conditions in individuals with a genetic predisposition. 


\section{Introduction}

In the mid-19th century, Louis Calmeil described a spontaneous arterial hemorrhage from the nose in the termination phase of migraine. Sacks [1], who reported the French psychiatrist's description, found migraine sufferers with engorged and purple turbinates. In the 1960s, Ikonomoff and Simeonoff [2] examined individuals with epistaxis and found that $71 \%$ of them were migraine sufferers, advocating that a relationship was mediated by the diencephalon. Children with migraine are more susceptible to epistaxis [3]. Topiramate, prescribed worldwide for migraine prophylaxis, may trigger violent nose bleeding [4]. Despite all these observations, only two cases of epistaxis associated with migraine without aura were described $[5,6]$.

Sporadic hemiplegic migraine (SHM) is a rare subtype of migraine with aura (MA). Diagnostic criteria require the presence of reversible motor deficits associated with at least one other transient neurological symptom, without similar episodes in relatives [7]. Hand paresis is practically universal in SHM, whereas the lower limbs and face are only affected in approximately $50 \%$ of all cases $[7,8]$.

\section{Case}

We describe a 47-year-old, right-handed male with a history of MA starting at the age of 20 . The episodes presented a stereotyped sequence beginning with a perception of vision through translucent water fountains, exponentially progressing from the periphery of the visual hemifield (usually the right one). A kinetic frame developed, consisting of fluttering and sparkling butterfly wings. The scintillating scotoma lasted $10 \mathrm{~min}$. A relentless frontal, bilateral, throbbing headache immediately developed, aggravated by head movements and accompanied by phono-photophobia and nausea.

The headache reached its maximum intensity at $90 \mathrm{~min}$, and an abundant epistaxis emerged from the right nostril with bright red blood, but the patient had no nasal pain. The headache intensity decreased immediately and was virtually absent within $15 \mathrm{~min}$. He experienced over 20 stereotyped episodes throughout his life. He also had episodes of migraine without bleeding and never presented epistaxis in other circumstances. No other spontaneous hemorrhages or ecchymosis have ever been observed. There were no nasal symptoms, arterial hypertension or analytical blood changes. He occasionally took paracetamol or nimesulide but never used salicylates, ergotamine, triptans, nasal vasoconstrictors or illegal drugs.

At the age of 35, he experienced a left scintillating scotoma, similar to previous ones. One hour later, during a short taxi ride, he felt a slight numbness in his left fingers and difficulty in verbal expression. When exiting the car, he fell to the ground. Motor dysphasia and left leg plegia were observed at the local hospital. No signs in the face or upper limb were observed. The crural weakness lasted for $2 \mathrm{~h}$. He was transferred to a central hospital to clarify the possibility of a stroke. Upon admission, he had a bifrontal, pressing headache with normal neurological examination. Another episode occurred 3 months later, beginning with a right scintillating scotoma followed by aphasia and right crural sensorimotor deficits. He had no headache. Twenty months later, he presented with a scintillating scotoma, aphasia and right brachiofacial plegia followed by a bifrontal throbbing headache. MRI (1.5 tesla) and blood tests were normal. Mutations in CACNA1A, ATP1A2, SCN1A and NOTCH3 were excluded. He maintained episodes of visual aura until the age of 39 . Afterwards, he stopped having auras or headache altogether. His parents and six brothers never experienced migraine or paroxysmal symptoms. His son, aged 23 years, has had side-shifting migraine with a scintillating scotoma since the age of 12 . 


\section{Discussion}

Three distinct aspects deserve our consideration: epistaxis, aura topography and natural history.

This is the third report of migraine-induced epistaxis but the first involving aura. Epistaxis appeared between the peak severity of headache and the beginning of its resolution, similar to previous descriptions $[5,6]$. Therefore, it could be considered as a symptom of the resolution phase of migraine. This chronological profile is consistent with one of the classical concepts of the resolution of migraine (lytic state or resolution by secretion) which may include vomiting, diuresis, lacrimation, sweating or epistaxis [1]. Co-occurrence of a throbbing headache and epistaxis could be due to the activation of the trigeminovascular system, causing vasodilation of dependent branches of the internal and external carotid arteries [6]. However, this attractive hypothesis presents an epidemiological weakness: migraine is common but descriptions of migraine with epistaxis are rare. The lack of similar cases reported is intriguing. The scarcity may be due to the depreciation of this symptom or the lack of a guided anamnesis.

Interestingly, descriptions of migraine sufferers with epistaxis can be found on nonscientific websites.

The inclusion of a specific question about epistaxis in the questionnaires of headache research centers would allow us to estimate the prevalence, timing and features of these episodes during migraine attacks, contributing to a better understanding of them.

The aura had a peculiar topography, inconsistent with the classical analytical neurological semiology. Motor aphasia was accompanied by weakness, even when it was in the left hand. The coexistence of aphasia and aura symptoms in the nondominant hand has been previously documented [8]. It is well known that hand paresis is practically universal in SHM, whereas the lower limbs and face are only affected in half of all cases [7, 9]. In this case, the lower limb was affected by weakness in the absence of deficits affecting the face or upper limb. The headache location was not contralateral to the aura, as previously described [10]. This set of evidence suggests that the spreading depression can initiate in a stereotyped manner in the occipital cortex (typical scintillating scotoma) and silently spread anteriorly or affect the cerebral cortex in an uneven manner. The medial frontal cortex (or subcortical structures) was affected in episodes of crural aura and primary somatosensory and motor areas of the convexity were relatively spared. These findings support the concept that pathophysiological phenomena of migraine are bilateral and pan-cerebral, regardless of the laterality of their capricious clinical manifestations.

The natural history of this disease has some peculiarities. It started with MA at the age of 20, and only 15 years later the patient exhibited three episodes of SHM followed by progressive remission (migraine swan song). It could be argued that this is a coincidence of two types of migraine. However, the scintillating scotoma, which is stereotyped and distinctive, is common to both MA and SHM episodes. Assuming the patient suffers from a single disease, the present case seems to demonstrate that the appearance of complex auras requires improbable interactions between environmental and endogenous conditions in individuals with a genetic predisposition. 
Interestingly, the patient's son had similar episodes of MA. Some asymptomatic relatives of SHM patients may be genetically affected but not subjected to strong enough triggers. It will be interesting to see if our diagnosis will evolve to familial hemiplegic migraine, which often occurs in 'sporadic' cases.

\section{References}

1 Sacks O: Migraine. Rev. and expanded. London, Picador, 1992, pp 13-33.

2 Ikonomoff SI, Simeonoff KR: New concepts on the diencephalic origin of epistaxis and migraine. Clinical observations (in French). Rev Neurol (Paris) 1968;119:229.

-3 Jarjour IT, Jarjour LK: Migraine and recurrent epistaxis in children. Pediatr Neurol 2005;33:94-97.

-4 Page RL, Bainbridge JL: Intractable epistaxis associated with topiramate administration. Ann Pharmacother 2006;40:1462-1465.

5 Sperber AD, Abarbanel JM: Migraine-induced epistaxis. Headache 1986;26:517-518.

-6 Durán-Ferreras E, Viguera J, Patrignani G, Martínez-Parra C: Epistaxis accompanying migraine attacks. Cephalalgia 2007;27:958-959.

7 Thomsen LL, Ostergaard E, Olesen J, Russell MB: Evidence for a separate type of migraine with aura: sporadic hemiplegic migraine. Neurology 2003;60:595-601.

$>8$ Peatfield RC, Gawel MJ, Rose FC: Asymmetry of the aura and pain in migraine. J Neurol Neurosurg Psychiatry 1981;44:846-848.

$\checkmark 9$ Russell MB, Olesen J: A nosographic analysis of the migraine aura in a general population. Brain 1996;119(Pt 2):355-361.

10 Khalil NM, Nicotra A, Wilkins AJ: Asymmetry of visual function in migraine with aura: correlation with lateralisation of headache and aura. Cephalalgia 2011;31:213-221. 